\title{
Measurements and geometry
}

\author{
Valery D. Mazin ${ }^{1}$ \\ ${ }^{1}$ Peter the Great St. Petersburg Polytechnic University, Russia
}

\begin{abstract}
The paper is aimed at demonstrating the points of contact between measurements and geometry, which is done by modelling the main elements of the measurement process by the elements of geometry. It is shown that the basic equation for measurements can be established from the expression of projective metric and represents its particular case. Commonly occurring groups of functional transformations of the measured value are listed. Nearly all of them are projective transformations, which have invariants and are useful if greater accuracy of measurements is desired. Some examples are given to demonstrate that real measurement transformations can be dealt with via fractional-linear approximations. It is shown that basic metrological and geometrical categories are related, and a concept of seeing a multitude of physical values as elements of an abstract geometric space is introduced. A system of units can be reasonably used as the basis of this space. Two tensors are introduced in the space. One of them (the affinor) describes the interactions within the physical object, the other (the metric tensor) establishes the summation rule on account of the random nature of components.
\end{abstract}

\section{Section: RESEARCH PAPER}

Keywords: measurements; geometry; projective metric; basic measurement equation; geometric space

Citation: Valery Mazin, Measurements and geometry, Acta IMEKO, vol. 10, no. 2, article 14, June 2021, identifier: IMEKO-ACTA-10 (2021)-02-14

Section Editor: Francesco Lamonaca, University of Calabria, Italy

Received April 2, 2021; In final form May 18, 2021; Published June 2021

Copyright: This is an open-access article distributed under the terms of the Creative Commons Attribution 3.0 License, which permits unrestricted use, distribution, and reproduction in any medium, provided the original author and source are credited.

Corresponding author: Valery D. Mazin, e-mail: masin@list.ru

\section{INTRODUCTION}

The purpose of this article is to define the commonalities between the concept of measurements and geometry; a method that uses the elements of geometry to model the basic elements of a measurement process.

Among many approaches that are used to describe fundamental measurement categories, geometrical approach is often underrated despite the fact that geometry as a science has originated from measurements and only later turned to a new higher level of generality. This paper attempts to argue that measurements and geometry are related, and geometry is not just another branch of math.

At all times the most prominent authorities in the scientific world have acknowledged the fundamental and special place that geometry takes in the system of exact sciences. Thus, Spinoza believed that it is geometry that "reveals a causal connection in nature". Newton said that "geometry expounds and justifies the art of measurement" [1]. In [2] we find Einstein's statement, according to which "geometry must precede physics, since the laws of the latter cannot be expressed without geometry. Therefore, geometry must be considered as a science, logically preceding every experience and every experimental science." A remarkable illustration of this thought is also presented in the book by B. Mandelbrot, "The Fractal Geometry of Nature" [3]. In "The Encyclopedia of Mathematics" [4], the special role of geometry is characterized in the following way: "Developments of geometry and its applications, advances in geometric perception of abstract objects in various areas of mathematics and natural science provide solid evidence of the importance of geometry as one of the most profound and fruitful means for cognizing reality" $[5,6]$.

Today measurement specialists rarely use geometrical apparatus both in general and particular cases (with exception of, maybe, measurements at the elementary level). Instead, analytical approach absolutely dominates the field. However, [7] highlights the huge heuristic value of geometric representation of the concepts of analysis; saying that geometry "is becoming increasingly important in ... physics. It simplifies mathematical formalism and deepens physical comprehension. This renaissance of geometry has had an impact not only on the special and general theory of relativity, obviously geometric in nature, but also on other branches of physics, where the geometry of more abstract spaces is replacing the geometry of physical space."

Today, no one seems to deny the fact that the science of measurements is actually a metascience, which is used in all natural and technical sciences, to say the least. For this reason, 
the apparatus has to be represented by disciplines with the same or higher order of generality. Geometry is just such a discipline.

Section 2 shows that the basic equation for measurements is a special case of the expression of projective metric. In Section 3 , functional measurement transformations are looked at in the context of groups theory. Section 4 is devoted to identifying the relationship between metrological and geometrical categories. The concluding section summarizes the main idea of the paper and points out on its practical usefulness.

\section{PROJECTIVE METRIC AND BASIC MEASUREMENT EQUATION}

The essence of any measurement has always been a comparison with a known unit. Among various geometric systems, the most common one is projective geometry, which, according to M. Komatsu [8], represents geometry as a whole. Projective geometry only studies the mutual relations between figures and in this sense is akin to measurements.

A segment of a numerical axis can traditionally represent the value of a measured quantity. In projective geometry, the distance between two points is determined using a Cayley metric (projective metric)

$$
l=c|\ln V|,
$$

where $c$ is the constant,

$$
V=\frac{x_{3}-x_{1}}{x_{2}-x_{3}} / \frac{x_{4}-x_{1}}{x_{2}-x_{4}}
$$

(complex, or double ratio of four points of a straight line), $x_{1}, x_{2}, x_{3}, x_{4}$ are the coordinates of the points on the line.

Let $c=1, x_{3}=0, x_{4}=\infty$. Then from the equations above it follows that

$$
l=\left|\ln \left(\frac{x_{1}}{x_{2}}\right)\right|=\left|\ln \left(\frac{x_{2}}{x_{1}}\right)\right|,
$$

hence

$$
\mathrm{e}^{l \cdot \operatorname{sgn}\left(x_{2}-x_{1}\right)}=\frac{x_{2}}{x_{1}}
$$

and

$$
x_{2}=x_{1} \cdot \mathrm{e}^{l \cdot \operatorname{sgn}\left(x_{2}-x_{1}\right)} .
$$

The meaning of the quantities in the last equation leaves no doubt that what we have here the "basic equation of measurements" usually written as

$$
x=\{x\}[x],
$$

where $x$ is the measured quantity,

$$
\{x\}=\mathrm{e}^{l \cdot \operatorname{sgn}\left(x_{2}-x_{1}\right)}
$$

is its numerical value, and $[x]$ is the quantity unit.

The latter is taken for granted and does not seem to require any proof. However, as we can see, it is deduced from the definition of projective metric, a fact that can hardly be accidental. Thus, it is worth mentioning a statement by famous mathematician Holder [9]: "To prevent misunderstanding, I note here that the axioms of the theory of quantities as they appear here should not be presumed in geometry or applied to segments and volumes. On the contrary, there are examples of purely geometric axioms for points and segments, from which it can later be proved ... that for segments there are facts that in general theory of measurable quantities are presupposed as axioms".

In connection to the above said, let us make the following remark: from (1) it follows that the logarithmic scale, widely used in measurements as well as in physics and technology, is nothing but a scale in projective metric. In general, the simple relations stated above suggest that there is a principled connection between the fundamental concepts of geometry and measurements. The basic equation for measurements, fundamental and meaningful in itself, happens to be a particular case of a fundamental geometric relationship too.

\section{GROUPS OF FUNCTIONAL MEASUREMENT TRANSFORMATIONS}

In addition, measurements and geometry are related by the fact that invariants are widely used in both disciplines. In measurements, this leads to improved accuracy. [10] shows an example of invariant principle applied to a simple ratio of three values of the measured quantity to the affine measurement transformation. In geometry, invariants generally have fundamental significance, since according to F. Klein's "Erlangen Program" [11], various geometries represent the invariant theories of the relevant transformation groups. It should be noted that the function of the channel transformation of measurement system, $y=\mathrm{f}(x)$, certainly belongs to one of the following groups (we do not mean the groups mentioned in the "Erlangen Program"):

$y=x$

is the identical group,

$y=x+\beta$

is the shift group,

$y=a \cdot x$

is the similarity group,

$y=a \cdot x+\beta$

is the affine (linear) group

$$
y=(\alpha \cdot x+\beta) /(\gamma \cdot x+\delta)
$$

is the projective (fractional-linear) group,

$$
y_{2} \geq y_{1} \text { by } x_{2} \geq x_{1} \text {, or } y_{2} \leq y_{1} \text { by } x_{2} \geq x_{1}
$$

is the group of monotonous transformations.

All these transformations, except for (13), have invariants. Such an invariant for (12), which includes all the previous groups (8) through (11), is a complex ratio of four points on a straight line $[12,13]$. For $(11)$, the invariant is the simple ratio of three points on a straight line $\left(x_{2}-x_{1}\right) /\left(x_{3}-x_{2}\right)$, for (9) and (8), except for the two indicated invariants, $x_{2}-x_{1}$, it is the usual Euclidean distance between two points lying on the coordinate axis.

The last two types of transformations are non-linear in general. If nonlinearity is small, then most commonly the corresponding experimental dependence can be satisfactorily approximated by a fractional-linear function [12], [14] - [16]. The remarkable property of the latter is that it belongs to the group of projective transformations while its form can vary a lot. Such a transformation can be visualized as an image of a projection of the input scale on the output scale. The group property is 


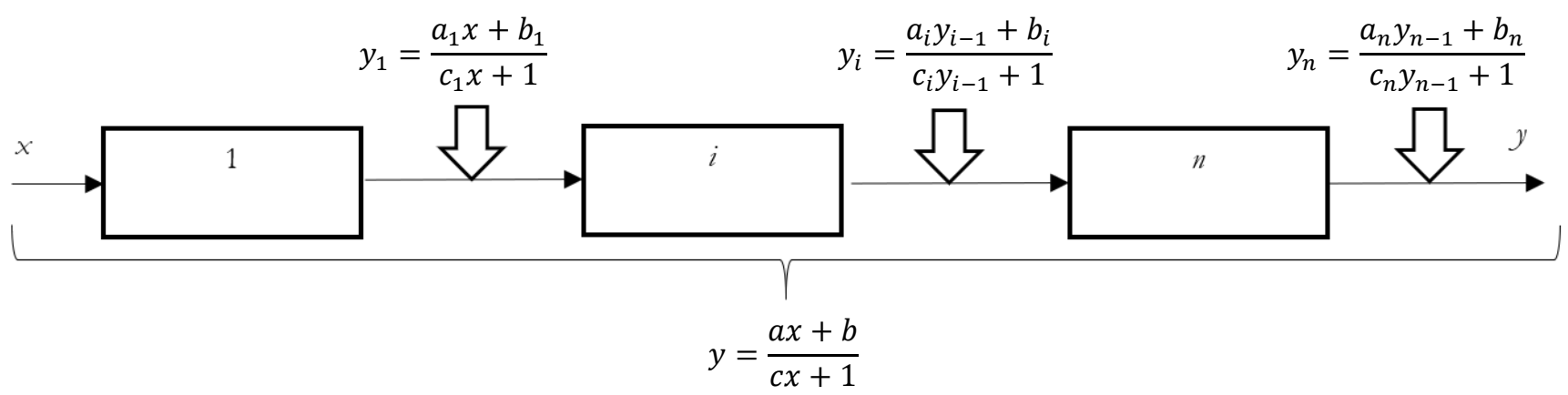

Figure 1. The circuit of fractional-linear transformations in a measuring channel.

expressed in the fact that the superposition of a series of fractional-linear functions is the same function and does not lead to higher complexity (Figure 1), while the inverse transformation is also fractional-linear. Thus, a unified mathematical description becomes possible both for the intermediate transformations in the channel and for the whole transformation.

For significantly nonlinear transformations or for improved accuracy of the approximation, it is advised that several fractional-linear functions should be used. They are either summed up or applied to sequential sections of the characteristic (piecewise approximation).

In the summation case the output value is obtained as the sum of the results of fractional-linear transformations. To make it possible, the following conditions must be met:

The result of the approximation by function

$$
y=Q(x) / P(x)=\sum_{0}^{m} a_{i} x^{i} / \sum_{0}^{n} b_{i} x^{i}
$$

has single roots in the denominator;

$m$ does not exceed $n$ by more than 1 .

If $y$ is a proper fraction, then it can be converted into a sum $\sum_{i=1}^{n} \frac{A_{i}}{\left(x-\alpha_{i}\right)}$,

where $\alpha_{1}, \ldots, \alpha_{n}$ are the roots of the denominator, the coefficients are found from the equation

$$
A_{i}=\frac{Q\left(\alpha_{i}\right)}{P^{\prime}\left(\alpha_{i}\right)}
$$

whereas

$$
P^{\prime}\left(\alpha_{i}\right)=\left.P^{\prime}(x)\right|_{x=\alpha_{i}} .
$$

If $m=n$, then a constant is added to the sum of the fractions as a result of extracting the integer part.

If $m=n+1$, then a linear function is added.

In both instances we deal with a particular case of a fractionallinear function.

If any the roots in the denominator are complex, nothing changes in principle, but some of the summable fractional-linear functions turn out to be complex. At the same time, all their remarkable properties are sustained, including the presence of an invariant - a complex relationship of four arbitrary points

$$
\frac{\left(x_{3}-x_{1}\right)}{\left(x_{2}-x_{3}\right)} / \frac{\left(x_{4}-x_{1}\right)}{\left(x_{2}-x_{4}\right)} \text {. }
$$

An example can be the results of approximation of the calibration characteristics of two temperature sensors. Let the first one be a platinum thermoresistor (its characteristic is utilized to model the international practical temperature scale). In the range of $-259^{\circ} \mathrm{C} \div+660{ }^{\circ} \mathrm{C}$, we obtain

$$
W=\frac{-2.244 \cdot 10^{4}}{t+2.768 \cdot 10^{3}}+\frac{2.925}{t+280.063}+\frac{-7.227 \cdot 10^{4}}{t-7.947 \cdot 10^{3}},
$$

where $W$ is the ratio of resistance at temperature $t$ in ${ }^{\circ} \mathrm{C}$ to resistance at zero Celsius. The standard uncertainty of this approximation is $0.7{ }^{\circ} \mathrm{C}$, which corresponds to $0.08 \%$ with respect to the temperature range and is considered acceptable for most practical cases. Let the second sensor be a $\mathrm{Pt} / \mathrm{Rh}$ thermocouple with $30 \% / 6 \%$ Rh content. In the range of $0{ }^{\circ} \mathrm{C}$ $\div 1800{ }^{\circ} \mathrm{C}$ its characteristic is approximated by expression

$$
\begin{aligned}
E= & \frac{-4.514 \cdot 10^{6}+5.287 \cdot 10^{7} i}{t-186.827-2.807 \cdot 10^{3} i} \\
& -\frac{4.514 \cdot 10^{6}+5.287 \cdot 10^{7} i}{t-186.827+2.807 \cdot 10^{3} i}-\frac{4.86 \cdot 10^{8}}{t-1.302 \cdot 10^{4}}
\end{aligned}
$$

and the standard uncertainty will equal to $0.3 \%$.

In the case of piecewise linear fractional approximation there are no restrictions with respect to accuracy (uncertainty), but it is more difficult to implement.

Whichever fractional-linear approximation case is chosen, the need for mathematical methods is limited to four arithmetic operations.

Using invariance fits the purpose of measurement, which is not about transformation, but rather about preserving the information. Indeed, in order to restore the characteristics of the original signal using measured characteristics of the converted signal, some kind of relationship between the signals has to be retained during the chosen transformation.

From this perspective, the measuring transducer should be called a transmitter rather than a transducer, i.e. in this case the name is not associated with the main property of an object but reflects its secondary property instead. This happens because the transfer and transformation of the quantity value (including scaled transformation, i.e. energy level transformation) correlate to each other in the same way as the essence and a phenomenon; in other words, a dualism takes place.

It is the transformation, not the transfer of the value that is visible to an observer. As in other similar cases, the object was named for its superficial, rather than essential property.

Ideally, in all types of measurement transformations such as the quantity type transformation, identity transformation, modulation and demodulation, the form of representing the transformation (e. g. analog-digital), code conversions, etc. the amount of information remains intact. In these transformations, errors mean the loss of information, and it is the degree of this loss, not the type of transformations that determines the quality of a measuring channel. 
Perhaps, the reason for the difficulty with the classification of measuring transducers that has still not been resolved is that all the variants of such a classification that are known so far are created on that side of the above-mentioned dualism that characterizes the phenomenon rather than the essence. In other words, what we are trying to do is classify the types of transformations; whereas what we should do is classify the types of information preservation.

This situation, which occurs while fractional-linear and their dependent transformations are used, is consistent with the general concept of the measurement procedure. The very basic procedures, for example, when the length was measured, consisted of two stages: the mutual displacement of the measured object and the measure, and their comparison with each other. Historically, this original essence of measurement is now perceived only by its second stage, while the first stage is actually no less significant.

It is worth highlighting that what in the early measurements was omnipresent mechanical displacements, nowadays is replaced by measurement transformations.

The analogies between displacements in an ordinary space and measurement transformations can be formalized even further. In length measurements, the correlation we wish to preserve is seen as the distance between the points, and this distance remains unchangeable no matter what the shifts and turns are. If we determine the distances in terms of the values that are preserved during such transformations for a set of all possible signals, we will arrive to the geometrical interpretation of a measurement procedure as a transformation that preserves the distance, i.e. as "displacements" in the relevant space. With fractional-linear transformations (for example, when using a voltage divider), complex ratio $V$ is preserved. But projective metric is thereby preserved too. Since fractional-linear transformations preserve projective metric, it is only natural to call them the "displacements in a projective space". At the same time, they are the most common among all the above transformations (except for monotonous transformations), each preserving the distance. It can be assumed that it is this property that permits us to either correct errors effectively or implement algorithms that eliminate errors in the first place.

In these terms, the stages of the measurement procedure consist of displacements in a projective space and comparison with a master reference, i.e. coincide with an ordinary length measurement procedure. A typical example is measuring voltage with a bitwise-balancing voltmeter, whose range is smaller than the voltage measured. In such a case the voltage is pre-attenuated by a divider. The divider produces the corresponding section of

Table 1. Correspondence between metrological and geometric categories.

\begin{tabular}{l|l}
\hline Metrological category & Geometric equivalent \\
\hline $\begin{array}{l}\text { An object, a measuring instrument } \\
\text { with deterministic relationships }\end{array}$ & $\begin{array}{l}\text { The space of affine connectivity, or a } \\
\text { Riemannian space }\end{array}$ \\
\hline Physical quantity & Point in the space, vector \\
\hline System of units & Basis \\
\hline $\begin{array}{l}\text { Probability characteristics and } \\
\text { statistical relationship of physical } \\
\text { quantities }\end{array}$ & $\begin{array}{l}\text { Metric tensor (determines the space } \\
\text { geometry) }\end{array}$ \\
\hline $\begin{array}{l}\text { Analog measurement } \\
\text { transformation }\end{array}$ & $\begin{array}{l}\text { Affinor (determines the relationship } \\
\text { of the vectors) }\end{array}$ \\
\hline Analog-to-digital conversion & Vectors subtraction \\
\hline $\begin{array}{l}\text { Preservation of the measurement } \\
\text { information }\end{array}$ & Invariance \\
\hline
\end{tabular}

the voltage scale on its output, preserving its length in the projective metric, while the voltmeter makes the comparison.

As a preliminary conclusion, we can state so far that: any functional measurement transformation belongs to the group of monotonous transformations;

the most common monotonous transformation is a fractional-linear variety that can describe projective correlations analytically and act as displacements (whereas the dimensions of the moving object are invariant).

\section{CORRESPONDENCE BETWEEN METROLOGICAL AND GEOMETRIC CATEGORIES}

Table 1 shows the mutual correspondence of the fundamental metrological and geometric categories. Any line in Table 1 can be used as a departure point for further research.

The concept of the space of affine connectivity takes up the first place in the table among geometrical concepts.

It represents diversity, in which the field of the connectivity object is defined. The term "diversity" generally needs to be defined; however, in this case we will skip that as its meaning is obvious. As we know, the connectivity object characterizes the point of diversity, in which a local benchmark (or affine benchmark, referring precisely to the given point) is defined. In turn, the affine benchmark is a combination of the point itself and the coordinate basis. The connectivity object gives as answer to the question about how the coordinates of an arbitrary vector change as it is displaced along a certain curve while preserving orientation. In the general case, the coordinates will indeed change, because as the vector is moving from one point to another, the local benchmark to which the vector is momentarily related, changes too.

The space of affine connectivity is poor in terms of properties, but it becomes richer once a metric is introduced in it by means of defining a metric tensor. Then the space becomes Riemannian, a space of curved vectors. Such vectors represent physical quantities that characterize a specific physical object [17]. For such a vector space, a system of units can serve as a basis since a unit of any quantity can be expressed via the basic units of the system.

A good example is a vector acceleration receiver on a moving ship. This receiver reads accelerations in an acoustic wave in water and its purpose is to identify the locations of the sources of noise. The main part of the receiver is shown in Figure 2. We see six flat piezoelectric plates at the outside edges rigidly connected to a pair of strings in its middle, each pair of strings being fixed on the frame and running in three mutually perpendicular directions. The inner edges of the piezoelectric plates are perpendicularly joined to the faces of a cubic inertial element.

When the frame experiences acceleration, the inertia force acts upon the cubical element, which can be componentized along the axes perpendicular to the planes of the piezoelectric plates. These componentized forces cause electrical charges. The axes perpendicular to the planes of the piezoelectric plates form a coordinate basis, and together with the center of gravity of the cubic element create a local benchmark.

As the ship moves and experiences pitching and rolling, the location and orientation in space of the local benchmark changes, whereas the direction of the vector of acceleration of water particles in the acoustic beam remains the same. As a result, the 


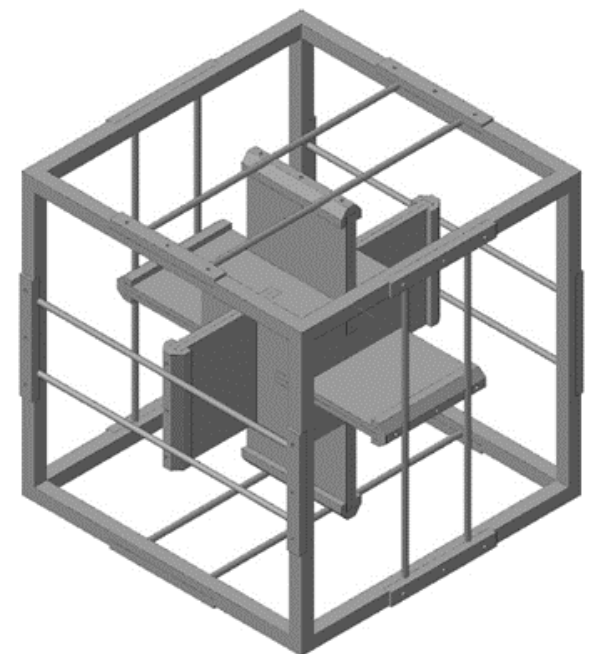

Figure 2. The main part of a vector acceleration receiver.

projections of this vector on the axis of the coordinate basis 1 change as determined by the connectivity object.

The connectivity object is a system of numbers, called connectivity coefficients. If each and every connectivity coefficient turns into zero, the diversity becomes an affine space. Vectors can be defined in it, so the space is a vector space. An affine space is a model of any particular object whose physical regular properties can be described by simple additive relationships.

The space describing a measuring instrument is generally multidimensional. In [18], we can see the application of multidimensional spaces apparatus.

In this space, the points and the vectors connecting them to the origin correspond to physical quantities. The basis of the space is the system of units.

An affine space can be identified for an object with any other kind of regular physical properties, but only in an infinitesimal region and with an accuracy no greater than that of the first order [19].

Let $y=f\left(x_{1}, \ldots, x_{n}\right)$ be any function of $n$ variables.

Then

$$
\mathrm{d} y=\left(\frac{\partial y}{\partial x_{i}}\right) \mathrm{d} x_{i}
$$

(implied summation over $i$ ) can be considered a vector, since coordinates $\left(\frac{\partial y}{\partial x_{i}}\right) \mathrm{d} x_{i}$ (at first approximation) are affine. The rule of adding the vectors, and, consequently, the space geometry, is determined by a metric tensor. Since the values for the generality should be considered random, the addition on rule must take into account their probability characteristics and statistical relationship. As shown in [20], if the coordinates for the vectors are expanded uncertainties, then the metric tensor is determined by the types of probability distribution, the coverage probability, the ratio of the terms, and mutual correlation. By today, the components of such a tensor for the most popular probability distributions and for 0.95 and 0.99 coverage probabilities have been determined by A. Chepushtanov [20]. If the coordinate system is formed by standard uncertainties, then the metric tensor is determined only by the mutual correlation.

The analog measurement transformation, which takes into account the design parameters of the device and influencing factors as input quantities, has a geometrical equivalent - affinor, the rule that states that each vector $\mathbf{d} \boldsymbol{x}$ is matched with a certain vector $\mathbf{d} \boldsymbol{y}$. The affinor is a square-matrix bivalent tensor.

Since the result of the analog-digital transformation is a number, it is only obvious that the quantity separates from the quality. In other words, the quantity rids of its physical carrier. Taking the logarithm of the basic equation for measurements yields two vectors - one for the numerical value and another for the unit. We do not touch here on the quasi uncertainty arising in the logarithm of a unit, we note only that it is a matter of logarithmising not the number „one”, but a unit of a physical quantity, which can have a different real meaning. Thus, in the logarithmic representation, the geometric essence of the analogdigital transformation is that the unit vector is subtracted from the full quantity vector, which is incidentally nothing else but computing how many units of the quantity (or which part of the unit) is included in the dimension of the quantity.

Finally, as it was stated above, the preservation of the measurement information characterizing the object conceptually corresponds to invariance. Information losses, inevitable in any measurement transformation (introduction of an uncertainty), means that this principle of correspondence is compromised.

When searching for the points of contact between modern geometry and measurements, special attention should be paid to non-Euclidean geometries. Riemann geometry is one of them, since its features are quite visible in the space of quantities. For instance, the ends of the logarithmic vectors of reciprocal quantities (such as resistance and conductivity) are located at the diametrically opposite points of a sphere that has its center at the origin of the coordinates. At the same time, there is practically no difference between them. They represent the same characteristic of the system. As it is known, the Riemann geometry is a spherical geometry with an additional condition of identification applied to the opposite points of the sphere. The obvious similarity between the physical and geometrical facts can hardly be accidental.

Moreover, there is evidence that different geometries work in case of different measurement ranges of the same physical quantities, which is only natural due to a wide generality of their geometric space. Further research in this area seems to be promising and new interesting results are expected.

Other promising applications of projective geometry are in explaining the laws of physics and image processing [22]. The basic concept here is projective mapping. It is most frequently used as a visual image rather than a mathematical structure. Being used in a strict geometric sense, this concept allows us to describe patterns with any level of complexity. Then the mapping parameters logically become variable, which is consequent to the change in the position of the projection center. This position, in turn, is affected by some physical causes, which now can be identified and explored.

Thus, the fundamental metrological categories have geometric equivalents. Problem defining in the area of measurements can be described using geometric terminology. Solving metrological problems seems possible if the powerful modern geometric apparatus is used.

\section{CONCLUSIONS}

The most important geometric concepts have equivalents in measurement theory. This knowledge allows us to apply geometric approach and apparatus to formulate a single 
mathematical description for important measurement categories, obtain new theoretical results, and model measuring procedures.

In particular, projective transformations can be used in such modelling. Due to their group properties, the characteristics of measuring devices can be described in a significantly simpler manner, whereas the present invariant allows us to increase the accuracy of measurements.

A model for any physical object, including the measuring device itself, can be represented as a vector space, whose elements, in turn, represent the quantities characterizing the object. This approach can be used in metrological analysis of measuring devices, where an important role is given to the summation of uncertainties. For such a summation in the geometric model, a metric tensor of the space is used, and in case of standard uncertainties such a tensor morphs into the coefficient of mutual correlation.

Thanks to the affinity of the concepts in geometry and measuring theory, measuring concepts and facts can be considered from a geometric standpoint and bring new interesting results.

\section{REFERENCES}

[1] I. I. Newton, Mathematical Beginnings of Natural Philosophy, University of California Press, 1999, ISBN 978- 0-520-08816-0.

[2] B. G. Kuznetsov, Einstein: life, death, immortality, Nauka, Moscow, 1980 [in Russian]

[3] B. Mandelbrot, Fractal Geometry of Nature, Computer Research Institute, Moscow, 2002 [in Russian]

[4] The Encyclopedia of Mathematics, Sovetskaia Entsiklopediia, Moscow, 1977 [in Russian]

[5] A. A. Penin, Analysis of electrical circuits with variable load regime parameters (Projective Geometry Method), Springer, Cham Heidelberg New York Dordrecht London, 2015, ISBN 978-3-31916351-2.

[6] A. S. T. Pires, A Brief Introduction to Topology and Differential Geometry in Condensed Matter Physics, Morgan \& Claypool, 2019, ISBN: 978-1-64327-371-6.

[7] B. F. Schutz, Geometrical Methods of Mathematical Physics, Cambridge University Press, Cambridge, 1980.

[8] M. Komatsu, Geometry Variety, Znanie, Moscow, 1981 [in Russian]

[9] O. L. Holder, Die Axiome der Quantität und die Lehre vom Maß: Ber. über die Verhandlungen der königlich sächsischen Ges. der wiss. mathem.-phys. Klasse, 1901, S. 1 - 65. [in German]
[10] E. M. Bromberg, K. L. Kulikovsky, Test methods for improving measurement accuracy, Energija, Moscow, 1978 [in Russian]

[11] F. C. Klein, A comparative review of recent researches in geometry: Bull. New York Math. Soc., N.Y., 1892-1893, pp. 215249.

[12] H. T. Nguyen, V. Y. Kreinovich, C. Baral, V. D. Mazin, Grouptheoretic approach as a general framework for sensors, neural networks, fuzzy control and genetic Boolean networks, 10-th IMEKO TC7 Internat. Symposium, Saint Petersburg, Russia, 30 June -2 July 2004, pp. 65-70. Online [Accessed 22 June 2021] https://www.imeko.org/publications/tc7-2004/IMEKO-TC72004-044.pdf

[13] I. N. Krotkov, V. Y. Kreinovich, V. D. Mazin, General form of measurement transformations which admit the computational methods of metrological analysis of measuring-testing and measuring-computing systems, Measurement Techniques 30 (1987), pp. 936-939. DOI: $10.1007 / \mathrm{BF} 00864981$

[14] O. A. Tsybulskii, Use of the complex ratio method in widerange measurement devices, Measurement Techniques 56 (2013), pp. 232-234.

DOI: $10.1007 / \mathrm{s} 11018-013-0185-2$

[15] O. A. Tsybulskii, The fractional-linear measurement equation, Measurement Techniques 60 (2017), pp. 443-450.

[16] O. A. Tsybulskii, Projective properties of wide-range measurements, Measurement Techniques 55 (2013), pp. 37-40. DOI: $\underline{10.1007 / \mathrm{s} 11018-013-0155-8}$

[17] V. D. Mazin, Physical quantity as a pseudo-Euclidean vector, Acta IMEKO 4(4) (2015), pp. 4-8. DOI: $10.21014 /$ acta imeko.v4i4.268

[18] B. V. Shebshaevich, P. P. Dmitriev, N.V. Ivantsevich et al, Network satellite radio navigation systems, Radio I Svyaz, Moscow, 1993 [in Russian].

[19] P. K. Rashevsky Riemannian Geometry and Tensor Analysis, Nauka, Moscow, 1967 [in Russian]

[20] V. D. Mazin, A. N. Chepushtanov, Application of a vectoranalytic model for metrological analysis of an infrared Fourier spectrometer, Measurement Techniques 51(2) (2008), pp. 152157. DOI: $10.1007 / \mathrm{s} 11018-008-9013-5$

[21] O. A. Tsybulskii, Analog-to-digital conversion with a hyperbolic scale, Metrology 12 (1990), pp.9-19 [in Russian]

[22] I. S. Gruzman, V. S. Kirichuk, V. P. Kosykh, G. I. Peretyagin, A. A. Spector, Digital image processing in information systems, Publishing House of the Novosibirsk State Technical University, Novosibirsk, 2002 [in Russian] 